\title{
Construction of Smart Campus System Based on Cloud Computing
}

\author{
Xiaofei Cheng ${ }^{1, a}{ }^{*}$, Rui Xue ${ }^{1, b}$ \\ ${ }^{1}$ Qingdao Huanghai College, China

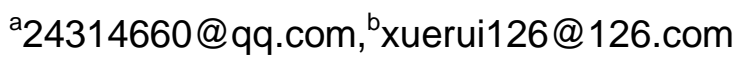

Keywords: Cloud Computing; Internet of Things; Smart Campus; Application

\begin{abstract}
In this paper, the authors stated the concept and characters of smart campus, and put forward a smart campus construction program based on cloud computing, internet of things and other technology. The analysis and study shows that the new information technologies are playing more and more important roles in improving education innovation. And the development of smart campus system based on information technologies will also improve management efficiency and decision-making levels of the managements of educational institutions.
\end{abstract}

Smart campus is a new idea in the development of information technology. It is a concrete practice of combination of the Cloud Computing, the Internet of Things and other technologies. It is also a kind of creativity of developing talent, improving management, and optimizing services. Cloud Computing is to integrate several of resources extensively, and offer supercomputing and storage capacity, which has three type of services: Infrastructure as a Service, Platform as a Service, Soft as a Service. The internet of things which divides into sensing layer, network layer and application layer is the product of effective fusion of sensor networks, Internet, and mobile communication network, and its core is the sensory system. The architecture of smart campus based on the cloud computing and the Internet of things consists of unified portal system, service support platform, data information convergence platform, network convergence platform, as well as information standard system and security maintenance.

\section{Introduction}

The rapid development of information technology has aroused teaching methods, great changes in the medium of instruction, teaching environment and teaching philosophy and other aspects of education, education, information technology is to achieve the modernization of education foundation. Our information technology education after 20 years of unremitting efforts, both hardware and software as well as environmental construction and other aspects have made great progress, but there are still many problems on the depth of integration of information technology in the teaching field of education, education, information technology development has entered a plateau. However, in today's network and promote information technology, has drawn worldwide pursuit of cloud computing and networking technology solutions for education information plateau problem brought hope and opportunity to inject new vitality, provides for educational innovation important support platform. Cloud-based computing and networking technologies wisdom campus construction is in full swing, the current field of education information has become the focus of attention.

\section{Cloud Computing}

Cloud Computing Concept. Cloud computing is a computing model based on the network, the use of non-local or remote server distributed computing, through the integration of grid computing, parallel computing, distributed computing, network storage, virtualization and load balancing technology, the many can be integrated into a computer to provide super-powerful computing and storage capacity of the system, and infrastructure as a service, platform as a service, software as a 
service model to achieve other commercial operation, allowing users to greatly saving investment and maintenance costs at the same time, quickly and easily share data and applications between different devices. $^{[1]}$

Cloud Computing Technology. Cloud computing platform for the wisdom of the campus provides a highly integrated, high efficiency, intelligent network data platform that web-based, server virtualization technology innovation, integration of various data resources to the resource pool, to provide users with a unified Press on-demand services. Cloud platform wisdom campus and include infrastructure services, platform services and software services. Infrastructure including servers, storage systems and network systems hardware platform as a service providing server rental virtual hardware resources and the like; internet services including authentication, authorization, data management, platform services system, mainly by Middleware, databases, and the development platform and other components; software services, is the core of the wisdom of the campus, but also the wisdom of the upper campus services, campus information system deployed in the layer, providing services through a unified portal, users get the service entrance. ${ }^{[2]}$ Cloud computing architecture is shown in Figure 1.

Unified portal system

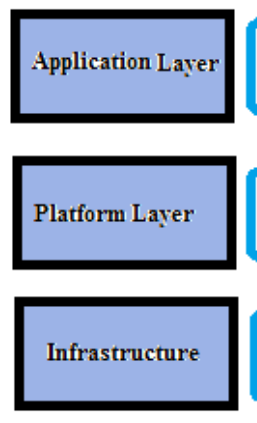

.Course platform;Learning platform

.Platform services, Authentication, Data management

System monitoring, Development platform

Cluster Management

Server, Network Management

Figure 1.Cloud computing architecture

\section{Networking Technology}

The Concept Of Things. MIT researchers have proposed the United States, things is through radio frequency identification technology, infrared sensors, global positioning systems, laser scanners and other information sensing device, according to the agreed protocol to anything connected with the Internet, information exchange and communication, in order to achieve intelligent identify, locate, track, monitor and manage a network. ${ }^{[3]}$

Things core technology of the Internet, efficient fusion of sensor networks and mobile communications networks, also a product of the physical system and efficient information systems integration. Wisdom campus construction process, Things perceptual system is an important part of the use of sensors, acquisition, RFID and sensing devices and other two-dimensional code, campus environment mark, wisdom campus environment to build a database, service platform, to effectively monitor the implementation of management and wisdom. The IOT sensing systems used in office automation, educational administration, learning systems, scientific research systems, financial systems, asset management, logistics management, library management, and so on. Things technology architecture has three layers, namely: perception layer, network layer and application layer. ${ }^{[4]}$ Internet of things technology architecture is shown in Figure 2. 


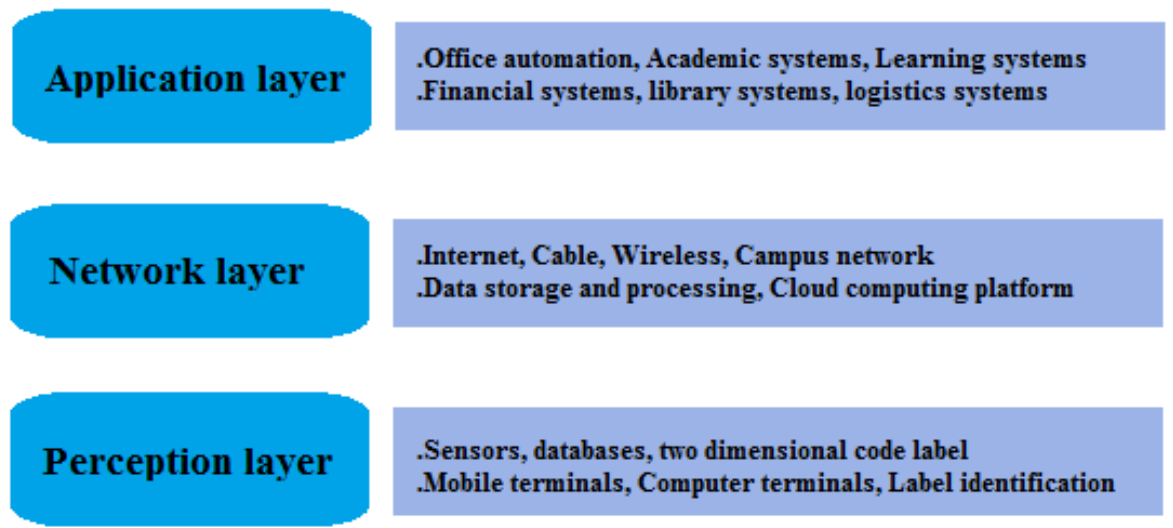

Figure 2.Internet of things technology architecture

\section{Smart Campus System Construction}

The Concept Of Smart Campus. The smart campus is to use modern information technology to cloud computing, virtualization, and things like changing the interaction of students, faculty, staff and campus resources, to improve the accuracy, flexibility and responsiveness of interactive applications, so as to enhance school effectiveness of the overall operation, service and smarter management of the campus model. Smart City thought leaders IBM's definition of the concept of the wisdom of the campus are: full use of teaching, research first-mover advantage and make full use of information and communications technology, from instrumented, integrated, intelligent starting to improve teaching and research level, to improve the school's own efficiency, effectiveness and efficiency of the management, enhance the influence of the school, realize the functions of education to serve the community, so that the wisdom of the campus to become an organic part of the wisdom of the city. ${ }^{[5]}$

The Smart Campus Architecture. Wisdom is the key in building a campus to break the traditional physical infrastructure and IT infrastructure in isolation from the inertia of thinking, that is, the data and information resources to a variety of applications for effective integration and integration, including the integration of the network, information orIntegration, service integration, according to intelligence-based management and personalized information services to integrate the data mode. According to the concept of wisdom campus, cloud-based computing and networking technology intelligence system architecture include unified campus portal services, service support platform, data integration platform, network integration services platform, as well as standard information system security and maintenance system. Smart Campus System architecture is shown in Figure 3.

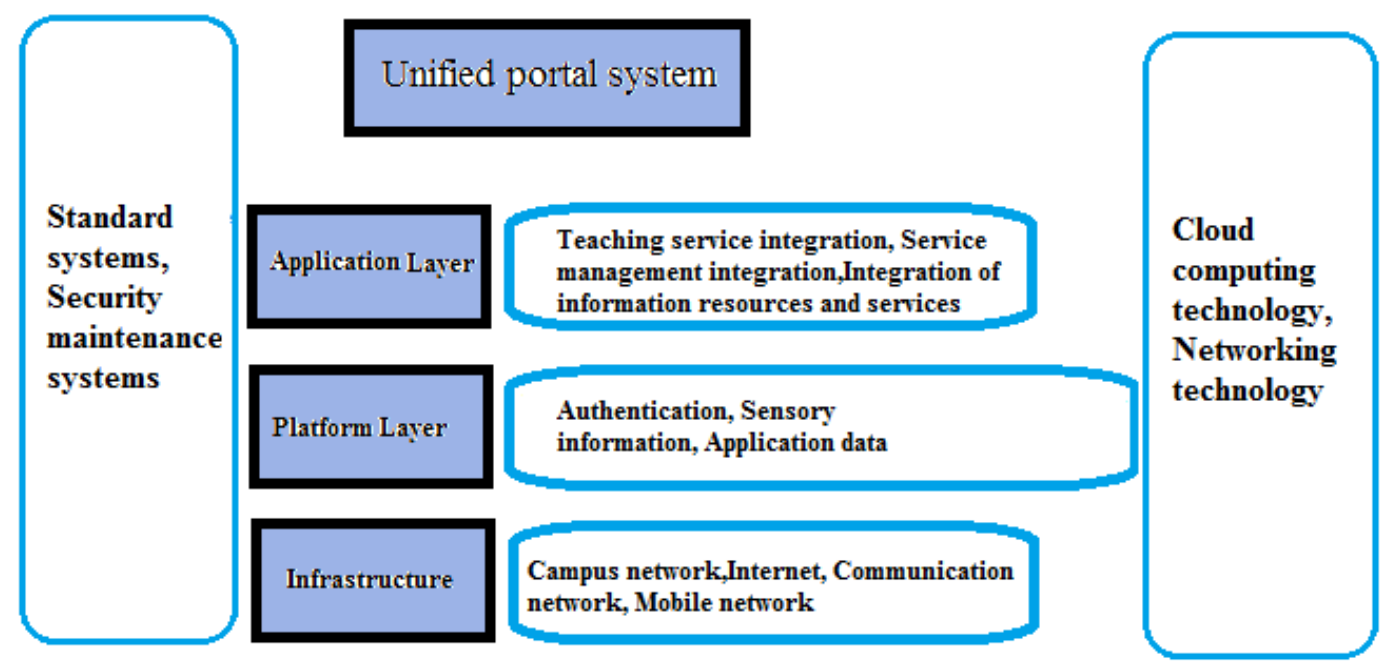

Figure 3. Smart Campus system architecture 
Unified Portal Service System. Unified portal service entrance system user access to services, unified portal system to access the system as a system framework, all subsystems are integrated through the portal system, the user's access is through the portal system unified authentication, unified login, whereby each sub permissions system. ${ }^{[6]}$

Service Support Platform. Service Support Platform is the purpose of the wisdom of the campus construction is based on a variety of applications to service processes, business processes and integrated information services as the core service integration architecture to achieve a variety of business interoperability and data sharing, to mention faculty for a full range of high-quality information services, improve the school systems of various application service capabilities and operational management capability, to achieve interconnection, sharing and collaboration.

Data Integration Platform. Data integration platform is the core wisdom campus, including the integration of information perception, identity and application data, etc., and the integration of data storage, middleware and software support, application integration ability to provide comprehensive information to the wisdom of Campus. ${ }^{[7]}$ Data integration platform is the role of integration through the restructuring, processing, management, data transformation and integration of resources, etc., unified information management, providing information services. Data fusion is the first platform to better serve the wisdom of campus infrastructure sharing teaching resources system, to provide students with a comprehensive and flexible teaching resources perception; second is for data exchange for the wisdom of the campus, data sharing and scientific management to provide comprehensive data and information services, namely, the school can be human, financial, material, activities and business processes perception, identification, tracking, analysis, processing and decision-making.

Based Converged Network Platform. Network convergence is the underlying platform hardware conditions wisdom campus construction, and laying the foundation for wisdom campus. Wired networks, wireless networks, the Internet and other technologies together to achieve a seamless connection between the various intelligence networks, comprehensive coverage, build a unified tube management, control and maintenance of network infrastructure platforms. ${ }^{[8]}$

Standard Information System

Information system is standard wisdom campus integration of various information first needs to establish a set of standards. Because there is no unified standard wisdom campus specifications, applications vary widely, it is difficult unified management of resources, resource publishing applications and resources, a standard system seems very important. Generally, wisdom campus system standard system construction from three aspects, one is to establish a complete information and data encoding standard system; the second is the use of international and national standards for software development; third is to establish wisdom campus hardware standards. ${ }^{[9]}$

Security Platform System Design. System security platform designed with cloud computing technology, the general security system consists of three layers, namely system-level security, network security level and application-level security components. A copy of a fault-tolerant multi-platform data, compute nodes isomorphism interchangeable and other measures to protect the data of high reliability, do not worry about viruses and data loss, and the cloud of rights management information data can be shared. ${ }^{[10]}$

\section{Summary}

Information technology continues to develop innovative education reform has brought new opportunities, based on cloud computing and networking technologies wisdom campus information technology in education is a very important part. In order to improve the quality of teaching, the promotion of education fair, improve management efficiency and decision-making levels play an important role. 


\section{References}

[1] Enji Sun, Xingkai Zhang, Zhongxue Li. The internet of things (IOT) and cloud computing (CC) based tailings dam monitoring and pre-alarm system in mines[J]. Safety Science,2012,504:.

[2Wu He, LidaXu. A state-of-the-art survey of cloud manufacturing[J]. International Journal of Computer Integrated Manufacturing,2015,283:

[3]K.E. Psannis, S. Xinogalos, A. Sifaleras. Convergence of Internet of things and mobile cloud computing[J]. Systems Science \& Control Engineering: An Open Access Journal,2014,21:

[4]ZhumingBi, David Cochran. Big data analytics with applications[J]. Journal of Management Analytics,2014,14:.

[5]Shiliang Luo, Lianglun Cheng, Bin Ren. Medical Monitoring and Managing Application of the Information Service Cloud System Based on Internet of Things[J]. Journal of Software,2014,97:.

[6]Drubin, Cliff. New IBM IoT Cloud Services to Drive Insights into Business Operations[J]. Microwave Journal,2015,585:.

[7]Abolfazli, Saeid,Sanaei, Zohreh, Bojanova, Irena. Internet of Cores[J]. IT Professional Magazine,2015,173:.

[8]Perera, Charith, Ranjan, Rajiv,Wang, Lizhe,Khan, Samee U. Big Data Privacy in the Internet of Things Era[J]. IT Professional Magazine,2015,173:.

[9] Lydon, Bill. Cloud computing[J]. InTech,2015,623:.

[10] McIlvride, Bob. SECURE REMOTE MONITORING and SUPERVISORY CONTROL[J]. Process Heating,2015,228:. 